\title{
PROJETO DE CONSTRUÇÃO DE UM MODELO FÍSICO EM ESCALA REDUZIDA PARA EXPERIMENTAÇÃO EM GEOFÍSICA DE POÇO.
}

\author{
Jéssica Pena Henriques ${ }^{a}$, Alberto Leandro de Melo ${ }^{a}$, Ana Paula de Souza Franco ${ }^{a}$
}

${ }^{a}$ Faculdade de Geofísica, Universidade Federal do Pará, Belém, Brasil.

Copyright 2016, SBGf - Sociedade Brasileira de Geofísica

Este texto foi preparado para a apresentação no VII Simpósio Brasileiro de Geofísica, Ouro Preto, 25 a 27 de outubro de 2016. Seu conteúdo foi revisado pelo Comitê Técnico do VII SimBGf, mas não necessariamente representa a opinião da SBGf ou de seus associados. É proibida a reprodução total ou parcial deste material para propósitos comerciais sem prévia autorização da SBGf.

\section{Resumo}

The analogical modeling proved very efficient in many areas of knowledge and, in particular, in Geophysics. From this effectiveness with the difficulty for understand certain concepts and procedures of Well Logging perceived by the author; this work proposes the development of a project to build a physical model with small-scale experiments in Well Logging. Introducing the basic concepts involving the exposed geological environment logging, logging itself and the difficulties in build a small-scale mode and the limitations. Developing a methodology of research from some analog modeling work until the conditions for model building through mainly by various types of tests. Which, after all, the model should be validated with the development of an electric probe also scaled.

\section{Introdução}

A modelagem analógica se mostrou muito eficiente em várias áreas do conhecimento e, em especial, na Geofísica. A partir dessa eficácia juntamente com a dificuldade de assimilar alguns conceitos e procedimentos da Geofísica de Poço percebidos pela autora, propõe-se neste trabalho o desenvolvimento de um projeto de construção de um modelo físico em escala reduzida para experimentação em Geofísica de Poço. Apresentando os conceitos básicos que envolvem tanto o ambiente geológico exposto a perfilagem de poço quanto a própria perfilagem até as dificuldades para a montagem de um modelo em escala reduzida, seu comprometimento com a teoria da similitude e suas limitações. Desenvolvendo uma metodologia que parte desde pesquisa de alguns trabalhos de modelagem analógica até a as condições para construção do modelo passando, principalmente, por testes de vários tipos. A qual, além de tudo, o modelo deve ser validado com o desenvolvimento de uma sonda elétrica também em escala reduzida.

\section{Metodologia/ Problema Investigado}

Ao projetar um modelo geológico para fins de perfilagem de geofísica de poço é necessário que todos os fatores que envolvam a construção sejam considerados para que a modelagem tenha validade. Fatores estes que vão desde a seleção do material sedimentar e de que forma simular os processos naturais até a aplicação do método geofísico que valide o modelo.
Portanto, dividiram-se as etapas do projeto de construção em tópicos: protótipo geológico, o modelo e a validação do modelo.

\section{Protótipo geológico}

Evidentemente neste projeto busca-se um modelo em escala reduzida. Sendo assim, é necessário, primeiro, basear-se em um protótipo geológico. No caso deste trabalho, deseja-se reproduzir ambientes sedimentares diversos contidos no mesmo modelo na tentativa de simular as propriedades físicas e analisar como são suas respostas geofísicas. Portanto, não foi usada como base uma sequência geológica real.

O ambiente geológico simulado consiste de formações, predominantemente, plano paralelas seguindo os três princípios que regem a organização de sequencias sedimentares propostas por Nicolau Steno: a superposição, a horizontalidade original e a continuidade lateral.

Para melhor entendimento da sequência estratigráfica, dividiu-se o modelo em três blocos: I, II e III. Sendo, o mais profundo, o bloco I, o bloco II intermediário e o bloco III o mais raso, como observado na Figura 1

Os registros destas camadas ficarão entre os extremos das linhas de argila e areia nos quais as amplitudes dependeram da fração de cada sedimento na camada. Seguindo na história geológica, houve a deposição de camadas de argila, de areia e da argila, respectivamente, registrando, também, as mudanças energéticas até chegar à camada mais superficial.

A escolha desse protótipo geológico, devidamente concebido, permitirá, também, que os métodos de prospecção geofísica aplicados no modelo possam ser avaliados quanto as suas capacidades de observação de certas estruturas.

Observação: no protótipo não foram incluídas dimensões métricas das camadas porque as dimensões verticais dependerão, exclusivamente, da escala utilizada no modelo.

\section{O Modelo}

O modelo segue exatamente a sequência estratigráfica do protótipo, porém em escala reduzida obedecendo à similitude geométrica. Assim, o modelo é uma cópia em escala reduzida do protótipo, toda via, alguns fatores são diferentes nos dois. Como visto anteriormente, para que haja similitude é necessário que o modelo e o protótipo sejam correlacionados através de uma transformação linear. Porém, quando se aplica uma transformação linear geométrica, todos os parâmetros geométricos devem ser 
igualmente transformados. No caso da transformação linear do protótipo para o modelo, o tamanho, a forma e a disposição dos grãos não serão devidamente consideradas. Apesar disto, a porosidade e a cimentação serão garantidas, para a reprodução das propriedades físicas e assim a teoria da similitude aplicada ao modelo é assegurada, pois as respostas geofísicas de interesses são mais influenciadas pelo comportamento macroscópico dos parâmetros petrofísicos.

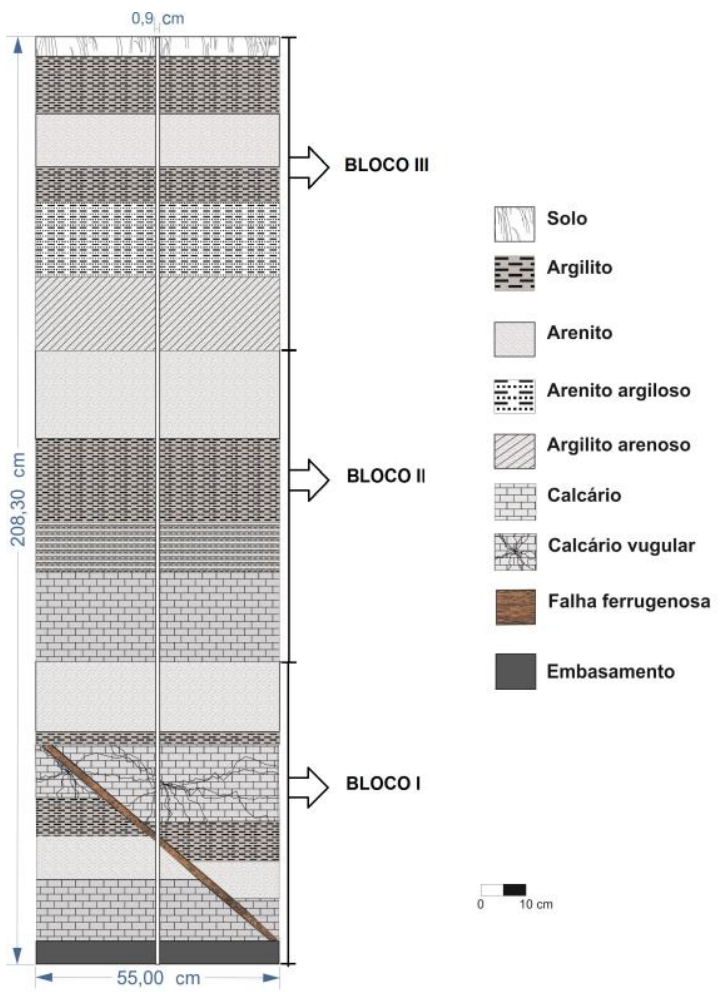

Figura 1: Sequência estratigráfica do modelo.

Para realizar a montagem do modelo dividiu-se a construção em etapas da seguinte forma: construção estrutural, construção do modelo e construção do poço.

\section{Construcão estrutural}

Neste projeto foi concebida uma estrutura metálica que possa conter o modelo físico em escala reduzida com dimensões razoáveis para experimentações com diversas técnicas de perfilagem de geofísica de poço. Para isso, pretende-se uma estrutura em ferro reforçada com dimensões $210 \times 61 \times 61 \mathrm{~cm}$ e com cavidades laterais de $4 \mathrm{~cm}$ para alocação das paredes de contenção que serão presas à estrutura metálica por parafusos como mostra a Figura 2

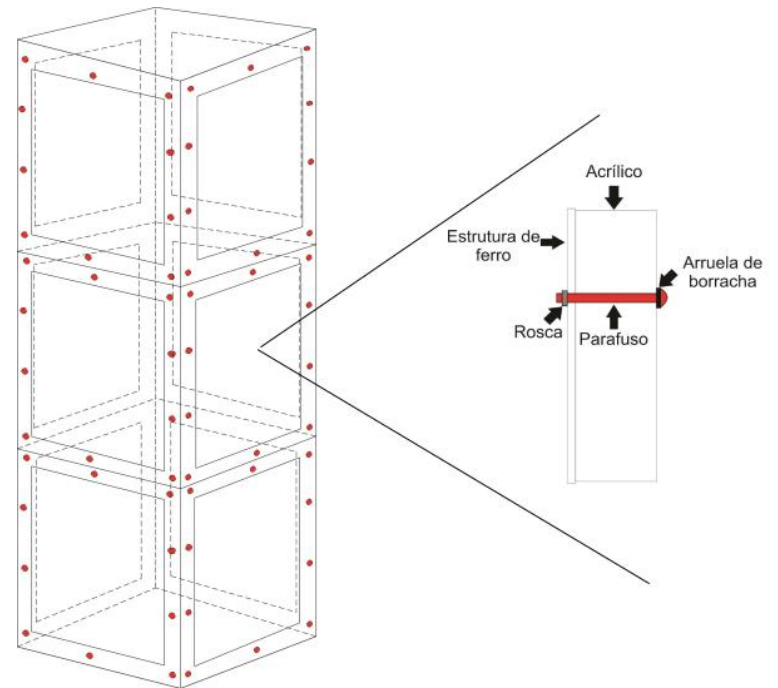

Figura 2. Esquema estrutura do modelo com detalhes da parede em acrílico.

A estrutura é projetada em três blocos devida necessidade de acesso à parte interna durante a construção das camadas de baixo para cima.

Para facilitar a construção do modelo, propõe-se a construção de bloco após bloco. Posteriormente a construção do bloco inferior às paredes de cada bloco serão colocadas e inicia-se a montagem das camadas do bloco e assim por diante. Idealmente é mais interessante que as paredes de contenção sejam de acrílico reforçado de $20 \mathrm{~mm}$, tal qual foi feita na experiência previa com a exposição "O que é Geofísica?"

Estas placas, como mostra o desenho esquemático da Figura 2, serão fixadas conforme a montagem das camadas, através de parafusos nas bordas coincidentes das placas e da estrutura metálica.

Acoplado às paredes laterais há um sistema de válvulas para permitir a injeção de fluido na falha e nas camadas permoporosas do modelo. A Figura 3 esquematiza o funcionamento desse sistema: uma torneira liga a parte exterior e interior do modelo. Na parte exterior a torneira está ligada a mangueiras que servirão para injetar o fluido ao distribuidor interno a camada, que consiste de um tubo de pvc de $1 / 2$ polegada furado posicionado dentro das camadas permoporosas o qual possibilitará a distribuição uniforme do fluído ao longo da camada. No outro extremo da camada, um absorvedouro (sumidouro) construído da mesma forma que o distribuidor possibilitará a retirada de fluido da camada. O uso das torneiras em ambas os lados do modelo permitirá o controle de pressão hidráulica nos poros das camadas. 


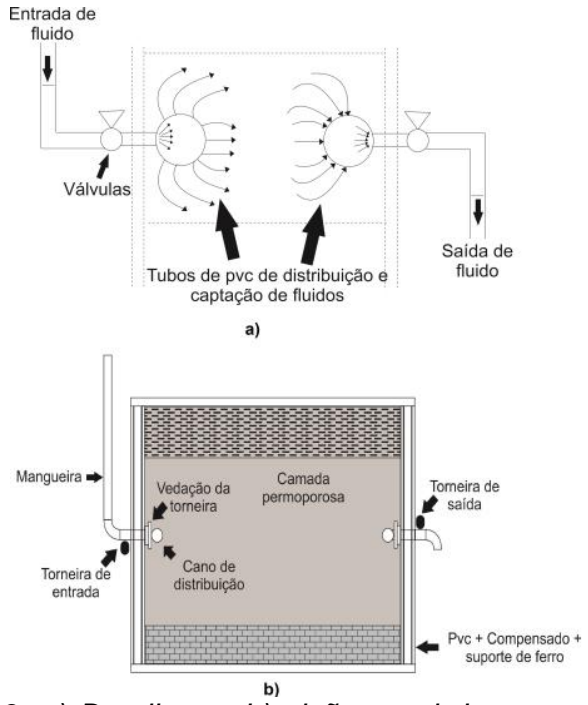

Figura 3: a) Detalhes e b) visão geral do mecanismo de injeção e retira de fluidos nas camadas permoporosas.

Ressaltando que os canos de distribuição estarão localizados na falha e nas camadas permoporosas.

Para evitar vazamentos é indispensável que todas as junções de paredes, os cantos, passagens para tubulações e qualquer caminho possível o qual a água possa passar sejam completamente vedados com material impermeável como, por exemplo, o silicone. É importante que a cola de vedação seja flexível, pois após o preenchimento total do tanque, com as camadas, as paredes laterais podem sofrer ligeiro afastamento e a cola poderá trincar, se for do tipo rígido. Após vedação, deve-se encher o aparato de água para garantir que a estrutura esteja completamente livre de vazamentos.

\section{Construção do modelo}

Como foi explicado anteriormente, devido o modelo simular um ambiente geológico para fins de perfilagem de poço foi adotado o uso de matéria prima natural como areia, argila e o calcário conforme o corpo estratigráfico já determinado.

Depois de selecionado o tipo de material, é importante considerar de que forma será depositado. Tendo em vista os princípios de Steno, são consideradas duas possibilidades: (a) deposição por precipitação em meio aquoso, e (b) deposição do material na forma de massa levemente consolidado. As duas opções tem suas vantagens e desvantagens.

A deposição controlada por precipitação do material é o que reproduz com mais fidelidade o modo que ocorre naturalmente em um regime de baixíssima energia. Em termos práticos essa forma também vem da experiência com os trabalhos anteriores ocorrendo da seguinte forma: enche-se o aparato com água e os sedimentos secos são adicionados, espalhando-se de forma uniforme por toda a área do tanque e, então, descerão por ação da gravidade e se acomodarão uniformemente, formando uma camada homogênea. Por ser um processo que se aproxima do natural, a porosidade, devido ao acomodamento, vai ser semelhante a um meio de pouca energia. Principalmente para a formação de argila essa possibilidade é a melhor, pois sua deposição será na forma laminada, o que é determinante para simular as propriedades físicas. Porém, a maior desvantagem deste processo é tempo para finalização da camada. Depois de colocado todo o material desejado é necessário esperar alguns dias para que a camada se estabeleça na melhor acomodação possível. Ao final, todo esse processo se torna muito mais longo.

Na segunda proposta, a construção da camada dar-se-á pela deposição direta da mistura de material levemente consolidado na forma de "massas" feitas de sedimentos, cimento e água. A mistura é depositada na estrutura e é moldada e compactada mecanicamente conforme 0 modelo geológico. Nas camadas de argila, esta alternativa afeta a sua distribuição laminar que depende da precipitação. Além disto, a mistura e moldagem mecânica a torna mais homogênea, provavelmente destruindo as características anisotrópicas própria da argila.

Independentemente da forma de deposição após a construção das camadas deve ocorrer o processo de maturação que, segundo Teixeira et al. (2000), é a diagênese que é composto por quatro etapas: compactação, dissolução, cimentação e recristalização diagenética. Destes, a compactação e a cimentação certamente ocorrerão. Os outros dois dependerão de fatores não controlados.

A compactação mecânica é o resultado da sobrecarga das camadas acima que produzirão uma redução de volume em profundidade. Como consequência, este processo levará a redução de porosidade e permeabilidade.

Para estimar quantitativamente os efeitos da sobrecarga é possível fazer um experimento simples que consiste em compactar uma amostra do mesmo tipo material referente à camada de interesse e submeter essa amostra a sobrecarga controlada em prensa mecânica. Posteriormente, analisar a amostra compactada obtendo sua porosidade e permeabilidade.

A dissolução é o efeito do fluído intersticial sobre as camadas. E, também, ocorrerá naturalmente no modelo. É de interesse que a dissolução seja a mínima para que não altere profundamente as camadas permoporosas.

No modelo, contudo, a cimentação não ocorrerá de forma completamente natural. Logo, a saída encontrada foi: adicionar cimento da construção civil aos sedimentos. A razão desta cimentação acelerada nos dois tipos de rochas presentes tem como principal motivo manter a integridade do poço.

Para alcançar a cimentação adequada, alguns testes foram realizados adicionando três tipos de materiais para acelerar a cimentação: cimento branco, cimento Portland e argamassa para assentamento de tijolo de vidro.

Os testes foram feitos com o mesmo tipo de sedimento mesmo tipo de grãos - e diversas porcentagens de cimento foram adicionados na mistura, com variação de 2 até $32 \%$ de massa total. 
Inicialmente a mistura do sedimento mais cimento foi analisada para apenas cada tipo de cimento separadamente. A partir disso, as conclusões foram de que o cimento branco e o Portland deram os melhores resultados quanto à rigidez e porosidade nos testes, além de diminuir a faixa de porcentagem ótima para 10 a $15 \%$. As amostras com argamassa mostraram-se muito friáveis para baixas concentrações e pouco porosas para as concentrações maiores.

Mas, foi a mistura dos dois que trouxe os melhores resultados: $90 \%$ sedimentos, $7 \%$ cimento Portland e $3 \%$ cimento branco em massa. As amostras ficaram rígidas, porém sem perder muito os valores de porosidades quando comparado com uma amostra $100 \%$ sedimento.

As medidas dos percentuais foram realizadas com uma balança semi-analítica da marca Shimadzu com precisão de $0,01 \mathrm{~g}$.

Para conhecer o quanto a cimentação afeta a porosidade, foram realizados alguns testes comparativos com e sem cimentação. Seguiram-se os seguintes procedimentos:

Para amostra não cimentada:

a)Tomou-se um determinado volume de água (Va) e mediu-se a massa daquele volume (mag).

b) Adicionou-se areia na água até que o volume ocupado pela areia (Var) fosse igual ao volume ocupado pela água anteriormente $(\mathrm{Va})$, portanto, $\mathrm{Vt}=\mathrm{Var}+\mathrm{Va}$ e mediu-se a massa total $(\mathrm{mt})$ correspondente a massa de água mais a massa de areia ( $\mathrm{mt}=\mathrm{mag}+\mathrm{mar})$.

c)Do volume total $(\mathrm{Vt})$ descartou-se o volume de água em excesso acima do volume de areia.

d)Mediu-se a massa map correspondente ao volume da areia saturada.

e)Calculou-se map-mar=mp, no qual $\mathrm{mp}$ corresponde a massa da água que ocupa os poros.

f)Considerando a densidade da água igual a $1 \mathrm{~g} / \mathrm{cm}^{3}$, conclui-se que $|\mathrm{mp}|=|\mathrm{Vp}|$.

g)Aplica-se a eq. 1 e obtém-se a porosidade.

Para a amostra cimentada:

a)Produziu-se a amostra ótima da misturados materiais e água.

b)Colocou-se a amostra em uma estufa durante dois dias para secar.

c)Após seca, mediu-se a massa da amostra mam e o volume Vam.

d)Saturou-se a amostra de água e a massa mt foi medida.

e)Calculando: $m t-m a m=m p$. $m p$ é a massa da água que ocupa os poros da amostra.

f) Usando o mesmo valor anterior de densidade da água, chega-se ao volume dos poros $\mathrm{Vp}$.
g)Aplica-se a eq. 1 e obtém-se o valor da porosidade.

Ao aplicar os testes, os valores de porosidade encontrados para amostra não cimentada foi de $33 \%$ e da amostra cimentada 23\%. Deve-se considerar que não houve sobrecarga extra para formação das camadas.

Apesar dos cuidados, alguns erros estão presentes nos procedimentos experimentais tais como: (1) o valor exato e preciso dos volumes e (2) a densidade da água utilizada. Para minimizar tais erros é necessário que haja total controle dos volumes medidos e a água usada no teste seja destilada garantindo o valor de densidade igual a $1 \mathrm{~g} / \mathrm{cm}^{3}$ e a temperatura conhecida.

Mesmo adicionando cimentação extra para dar alguma consolidação às rochas, os movimentos de acomodação dos sedimentos permanecem. Um desses movimentos é o escorregamento lateral, que é previsto pelo princípio da horizontalidade original citado anteriormente, pois para manter a forma horizontal da camada os sedimentos que estão mais altos em relação à linha média do topo livre da camada tendem a escorregar lateralmente.

Ainda que o movimento lateral dos sedimentos seja pequeno quando comparados com a estrutura de contenção do modelo, o movimento de milhares de grãos de areia pode provocar aumento de pressão significativo nas paredes internas do modelo. Para reduzir o escorregamento e, portanto, reduzir a pressão nas paredes da base, ao construir as camadas deve-se colocar um tecido bem fino em intervalos de alturas constantes. O uso do tecido tem por função aumentar o atrito horizontal e assim reduzir o grau de liberdade de movimentação dos grãos em contato com ele. O tecido deve ser sintético, de trama fina, colocado paralelamente à camada para que não prejudique a movimentação do fluido no sentido vertical.

É importante que, ao construir o modelo, amostras de todas as camadas sejam devidamente guardadas para posteriores testes experimentais com a finalidade de determinar as propriedades petrofísicas das amostras, quando submetidas a sobrecarga equivalente à sua posição no modelo e possível correlação com os perfis geofísicos que serão obtidos.

\section{Construção do poço}

O poço, um dos elementos mais importantes, deve atravessar todas as camadas sobrepostas que formam o modelo. Deve ser devidamente centralizado, vertical e estável. Para o modelo proposto adotou-se um poço com diâmetro de $9 \mathrm{~mm}$, suficiente para a introdução de diversos tipos de ferramentas geofísicas em escala reduzida.

É oportuno considerar que ao adotar o diâmetro de poço em $9 \mathrm{~mm}$ para o modelo, significa dizer que a escalabilidade perfeita para um poço protótipo de 8" terá como fator de escala perfeita de $=22,6$ e para um poço protótipo de 22" a escala perfeita será de $=62,2$. Contudo, o fator de escala deverá ser definido pela sonda utilizada na experimentação segundo seus aspectos teóricos. Assim, o fator de escala escolhido poderá não satisfazer a similitude perfeita para poços do protótipo 
com medidas 8" e 22", que são os padrões, devendo, então, o diâmetro do poço protótipo ser ajustado.

O poço do modelo deve ser preferencialmente do tipo open hole que garanta uma simulação mais próxima do real. Contudo, devem obedecer duas importantes necessidades: primeiro ter a garantia de que o poço seja estável por toda vida útil do modelo e segundo garantir que os campos aplicados e/ou observados não sejam profundamente afetados por possíveis revestimentos de sustentação das paredes do poço.

Para o caso do poço construído concomitante às camadas, precisa-se de um tubo que servirá de molde para o poço. O tubo deve ser rígido o suficiente para suportar a pressão radial das camadas em volta. Para isso, pode ser de cobre com diâmetro $7 \mathrm{~mm}$ e para evitar adesão de material na parede do tubo é importante que haja uma cobertura de material gelatinoso e solúvel em água como, por exemplo, sabão de coco. Esta cobertura é necessária para permitir a completa retirada do tubo posterior à construção das camadas. A cada camada construída o tubo deverá ser movimentado com um giro de tal forma a garantir que o mesmo não fique preso as camadas.

Após a construção completa, o tubo deverá se retirado por completo e será introduzida uma broca com lama de perfuração para eliminar o excesso de sabão e formação do mud cake e das zonas invadidas. Tendo, assim, a simulação mais fiel e segura possível da abertura do poço.

\section{Validação do modelo}

Para avaliar e validar o modelo geológico apresentado neste trabalho é necessário o auxílio de uma sonda que deve ser construída em escala reduzida, em correspondência com uma sonda geofísica real relacionada através de um fator geométrico ( ). Para a definição do fator geométrico devem ser levados em consideração os limites físicos impostos (dimensão lateral do modelo e diâmetro do poço).

\section{Sonda}

Uma sonda elétrica é proposta como uma ferramenta geofísica adequada para avaliar e validar o modelo em escala reduzida. Para isso, propõe-se uma cópia em escala reduzida da sonda elétrica ELXG, manufaturada pela Robertson Geologing Ltda. Está sonda é composta por um conjunto de ferramentas destinadas as medidas de resistividade elétrica, potencial espontâneo e raios gama. A sonda real possui 2,60 metros de comprimento, ao longo da qual estão distribuídos os eletrodos SPR (single point resistence), SHN (short normal, 16"), LN (long normal, 64"), SP (spontaneous potential) e um sensor GR (gamma ray). Um cabo elétrico com reforço de aço conecta a sonda ao console de superfície que é responsável pelo processamento dos dados enviados pela sonda deslocada no interior do poço. Um sistema de leitura de profundidade posicionado na boca do poço observa a posição da sonda em profundidade. Está posição, por sua vez, é controlada por um guincho mecânico. O console é, então, conectado ao computador para a aquisição e visualização dos dados por intermédio de um software (Winloger) proprietário da Robertson Geologing.

Neste trabalho serão reproduzidos apenas os eletrodos de resistividade e de potencial espontâneo em correspondência à sonda ELXG. Conforme já foi mencionado, o fator de escala geométrico aplicado ao modelo é controlado pela escala da sonda. Portanto, para o modelo representar um protótipo com camadas finas ou espessas, basta modificar a escala da sonda. Por outro lado, os limites físicos impostos ao modelo devem ser observados.

O tamanho da sonda elétrica de modelo correlacionada com a sonda ELXG por um fator de escala ( ) será limitada entre dois extremos: (1) para a simulação da sonda de 64" (M64), as linhas de campo geradas pela sonda modelo, na região iluminada, não devem sofrer fortes distorções devido à presença da parede do modelo, e, (2) para a simulação da sonda de 16" (M16), a distância entre os eletrodos de corrente (C1) e de potencial de 16" (P1), deve variar entre os limites 0,75 até 2 vezes o diâmetro do poço.

Para obedecer ao primeiro ponto, é recomendável que a sonda modelo M64 não ultrapasse $50 \%$ do raio diametral do modelo. Como o modelo proposto terá uma lateral de $55 \mathrm{~cm}$, é recomendável que a sonda modelo M64 não ultrapasse a $27,5 \mathrm{~cm}$ de espaçamento entre os eletrodos de corrente (C1) e de potencial (P1). Assim os efeitos de borda do modelo serão reduzidos.

Para obedecer ao segundo ponto, o afastamento entre o eletrodo de corrente e de potencial M16 não deve ser inferior 0,5 diâmetro do poço, pois, caso isso aconteça, a observação ficará basicamente restrita no interior do poço, e o método não será reproduzido fielmente.

Os dados do eletrodo SPR, embora possa ser reproduzido, obtidos não serão fieis ao caso real, pois sofrerão os efeitos de sonda devido à dimensão limitada do modelo e por isso produzindo fortes distorções na linha de campo.

Em consideração às condições acima, a sonda modelo deverá fazer parte de um conjunto instrumental cuidadosamente implementado, que juntos permitirão a medição dos parâmetros geofísicos em profundidade no interior do poço.

Para construir uma sonda multi-eletrodo, um cabo fino multicondutor deve estar disponível, pois cada eletrodo disposto na sonda deve ser conectado a um dos condutores do cabo. Na ausência de um cabo fino multicondutor, um cabo simples do tipo utilizado nos headphones poderá ser utilizado como cabo de sonda. Neste caso, a sonda devera ser apenas dois eletrodos e o afastamento deles deverá ser ajustado a cada descida da sonda no poço para realizar os dois tipos de medidas - normal curta e normal longa.

A sonda é a parte mais simples dentro do conjunto instrumental da perfilagem elétrica na modelagem. Ela consiste de simples eletrodos passivos colineares isolados eletricamente um do outro, com os 
distanciamentos dados pelo fator de escala protótipo/modelo. $O$ eletrodo de SP deve ser cuidadosamente coberto com negro de platina, ou chumbo, para evitar os efeitos de eletrodo. Os demais eletrodos podem ser construídos de aço ou de chumbo.

O cabo elétrico deve ser do tipo multicondutor (quando possível), bem isolado, de espessura mínima e com alguma resistência à atração, pois além de conduzir os sinais elétricos da sonda até a superfície, ele também servirá de cabo de transporte de sonda.

Um mecanismo de polia deve ser instalado na boca do poço para guiar o cabo de sonda durante a descida e subida. A polia deve ser conectada a um motor de baixa rotação (step motor) ou a um servo motor para a realização do trabalho de movimentação da sonda no interior do poço.

Um subsistema codificador angular deve ser acoplado, também a polia, para determinação da sonda em profundidade. O codificador deve ser capaz de gerar informações da direção e profundidade da sonda.

Para minimizar a construção de circuito eletrônica uma placa de aquisição de dados comercial NI MyDaq microprocessada, produzida pela National Instruments, destinada à área de automação, controle e aquisição de dado é adequada para ser utilizado com o sub sistema de aquisição de dados de sonda, controle de motor de guincho e leitura de posição da sonda. Esta placa contém conversores analógicos-digitais e portas de controle I/O necessários ao sistema instrumental de modelagem.

A comunicação com um computador de controle geral é feita via interface USB. Um notebook deve ser utilizado para controle da placa NI MyDaq. O software Labview, previamente instalado no notebook, permitirá desenvolver toda a programação necessária que consiste de todos os procedimentos de controle, aquisição de dados, processamento digital dos dados e geração dos perfis de resistividade e de potencial espontâneo.

Geração do sinal de corrente - para energizar o meio no modelo geológico por imposição de um campo elétrico entre os eletrodos de corrente na sonda e na superfície é necessário fornecer energia ao sistema. Embora, toda a teoria do método elétrico ate aqui foi desenvolvida utilizando uma fonte de energia de corrente continua, na prática, contudo, não se deve utilizar corrente continuas para evitar os efeitos de polarização de eletrodos. Para isso, faz-se uso de um sinal de corrente que alterna periodicamente, geralmente em torno de uns $100 \mathrm{~Hz}$, assim os eletrodos de corrente trocam de polaridade alternadamente evitando os efeitos da polarização de eletrodo. A placa NI MyDaq é capaz de gerar os sinais de corrente necessária, porém um amplificador de áudio é requerido para fornecer potencia suficiente para energizar adequadamente. Assim uma corrente elétrica fluirá entre os eletrodos deslocando os íons no interior do modelo.

Sinal de potencial elétrico - Uma vez energizado o modelo, em regime estacionário, os íons em movimento produzirão as linhas de corrente, que, por sua vez, estabelecerão as superfícies equipotenciais. Os eletrodos de sonda localizados em posições pré estabelecidos podem "sentir" estas superfícies equipotenciais. Dessa forma, a diferença de potencial entre os eletrodos na sonda e o outro fixado na superfície, pode ser medida. A ddp medida ( ) refletirá os efeitos da distribuição espacial da resistividade dos sedimentos acamadas no modelo.

É importante frisar que os eletrodos são do tipo passivo, conectados a um longo cabo elétrico e por isso podem ocorrer acoplamento capacitivos indesejáveis. Para evitar isto, é recomendável que um pré-amplificador casador de impedância seja utilizado e localizado na própria sonda.

O sinal de SP incorporado ao sinal de potencial medido deve ser obtido por processamento digital é o mais adequando, pois envolve menos custo instrumental. Considerando que a corrente injetada no meio é do tipo pulsada com ou sem retorno ao zero (NRZ), o valor do sinal SP nada mais será que o valor médio de medida no eletrodo M16.

Procedimentos matemáticos simples podem ser aplicados para a determinação de $\Delta V_{16}$ e o valor de SP. Deve-se lembrar de que o efeito SP esta presente em todo tipo de medida de diferença de potencial, pois é um efeito eletrolítico inerente a medida.

A corrente $I$, deve ser medida continuamente evitando que qualquer flutuação na corrente gerada possa induzir erros, o que pode acontecer se o procedimento for medir a corrente uma única vez.

A placa $\mathrm{NI}$ MyDaq tem canal $\mathrm{A} / \mathrm{D}$ suficiente para as medidas de $I, \Delta V_{16}$ e $\Delta V_{64}$.

Com estas medidas e as devidas filtragens o valor da resistividade $\rho_{a_{16}}$ e $\rho_{a_{64}}$ podem ser determinados por:

$\rho_{a_{16}}=k_{16} \frac{\Delta V_{16}}{I}$ e $\rho_{a 64}=k_{64} \frac{\Delta V_{64}}{I}$

Os dados obtidos devem ser plotados versus a profundidade obtida pelo codificador angular. Por fim 0 perfil geofísico pode ser obtido.

\section{Resultados esperados}

Embora este trabalho seja um projeto, portanto ainda sem dados reais, há como prevê-los de tal maneira que o modelo seja válido. Para isto, a resposta do modelo será perfil elétrico semelhante a qualquer um que sairia de uma perfilagem elétrica nas mesmas condições do protótipo.

A Figura 4 exibe um perfil imaginado a partir da observação de outros perfis reais com camadas semelhantes às descritas no protótipo que pode ser resultado da aplicação elétrica no modelo. Vale enfatizar que esse resultado esperado é estimado, podendo mudar em amplitudes, devido, principalmente, as resistividades dos fluidos que poderão ser injetados (fluidos de formação) e da lama de perfuração. No caso do perfil de 
ER a lama de perfuração é, provavelmente, mais resistiva que o fluido de formação o que explica as maiores amplitudes no levantamento de 16".

Ainda considerando a Figura 4 e baseando-se no conhecimento de perfis reais, pode-se presumir que as camadas mais próximas à superfície, provavelmente, terão medidas de ER distorcidas em relação às medidas de profundidade, pois a perfilagem se comportará como um levantamento em semi-espaço livre.

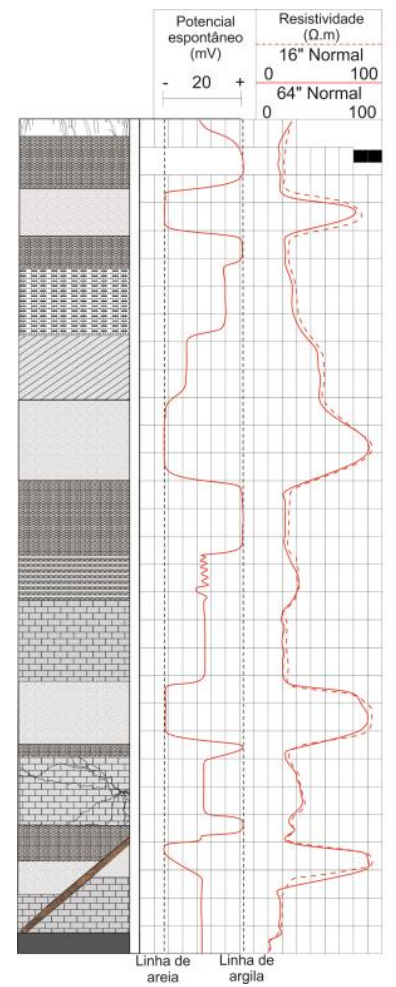

Figura 4: Perfil de potencial espontâneo e eletrorresistividade esperado para o modelo.

\section{Discussão e Conclusões}

Este trabalho apresentou uma maneira viável de construção um modelo físico em escala reduzida para aplicação da perfilagem de geofísica de poço. Depois de construído o modelo poderá ser usado como um laboratório didático para melhor explicar como ocorre uma perfilagem real, além disto, poderá ser utilizado para testes de outras ferramentas aplicadas em poço.

Considerando que este projeto honra as premissas da modelagem analógica, espera-se que 0 modelo construído comporte-se de forma similar ao caso real, com poucas diferenças. Pois a escolha de materiais naturais, tais aqueles encontrados em ambientes geológicos reais, permitirá que o conjunto de modelagem (modelo e sonda) gere dados com alto grau de fidelidade para fins didáticos e de testes.

Tendo em vista todo o embasamento teórico, materiais possíveis de utilização, processos de montagem e aplicação do método, conclui-se que a montagem do modelo e o uso da modelagem analógica para perfilagem de poço são viáveis.
A ferramenta proposta para validação do modelo é elétrica. Entretanto, recomenda-se que outras ferramentas sejam produzidas e testadas. Ainda com aplicação elétrica, poderão ser desenvolvidas ferramentas de microrresistividade e lateral log. Além destas, outras ferramentas poderão ser produzidas, tais como: sônicas, de indução (eletromagnéticas), de temperatura e magnéticas, sendo esta última mais voltada à mineração. Com ressalva apenas para uso da ferramenta radiométrica, que embora seja de extrema importância na geofísica de posso, pois, na escala reduzida deste projeto, sua construção torna-se difícil devido a indisponibilidade de sensores radiométricas, com boa sensibilidade, com diâmetros inferiores ao do poço do modelo.

Espera-se que este projeto possa estimular a construção do modelo e que este seja aplicado para a difusão de conhecimentos sobre a geofísica de poço.

\section{Referências}

Ellis, D. V.; Singer, J. M. Well logging for earth scientists. 1. ed. Nova York: Elsevier, 1987.

Girão Nery, G. Petrofísica. In: GIRÃO NERY, G. Perfilagem geofísica. HYDROLOG Serviços de Perfilagens Ltda, 2004. p. 1-19.

Pena Henriques, J. Projeto de construção de um modelo físico em escala reduzida para experimentação em geofísica de poço. 2014, 89 f. Trabalho de conclusão de curso - UFPA, IG, 2014.

Kearey, P.; Brooks, M.; Hill, I. Geofísica de exploração. São Paulo: Oficina de Textos, 2009.

Lowrie, W.; West, G. F. The effect of a conducting overburden on electromagnetic prospecting measurements. Geophysics, v.30, n.4, p.624-632. 1965.

Melo, A. L. Desenvolvimento de um conjunto de modelagem de alta resolução para métodos eletromagnéticos indutivos. 2004, 101 f. Dissertação (Mestrado em Geofísica) - UFPA, IG, 2004.

Salvadoretti, P. Construção de um modelo experimental simulando condições de perfilagem de indução. 1990, 54 f. Dissertação (Mestrado em Geofísica) - UFPA, IG, 1990.

Silva, N. Estudo do sinal elétrico de potencial espontâneo associado ao fluxo de água em meios porosos. 2011, 98 f. Tese (Doutorado em Geofísica) - Universidade de São Paulo, Instituto de Astronomia Geofísica e Ciências Atmosféricas, 2011.

Teixeira, W.; et al(Orgs.) Decifrando a terra. São Paulo: Oficina de Textos, 2000

Telford, W. M.; Geldart, L., P.; Sheriff, R. E. Applied geophysics. 2 ed. Cambridge: Cambridge University Press. 1990. 